\title{
Poincaré Bifurcations of Two Classes of Polynomial Systems
}

\author{
Jing Wang and Shuliang Shui \\ College of Mathematics, Physics and Information Engineering, Zhejiang Normal University, Jinhua 321004, China
}

Correspondence should be addressed to Shuliang Shui; shuisl@zjnu.cn

Received 24 May 2013; Accepted 27 June 2013

Academic Editor: Luca Guerrini

Copyright (C) 2013 J. Wang and S. Shui. This is an open access article distributed under the Creative Commons Attribution License, which permits unrestricted use, distribution, and reproduction in any medium, provided the original work is properly cited.

Using bifurcation methods and the Abelian integral, we investigate the number of the limit cycles that bifurcate from the period annulus of the singular point when we perturb the planar ordinary differential equations of the form $\dot{x}=-y C(x, y), \dot{y}=x C(x, y)$ with an arbitrary polynomial vector field, where $C(x, y)=1-x^{3}$ or $C(x, y)=1-x^{4}$.

\section{Introduction and Main Results}

In the qualitative theory of real planar differential systems, one of the main problems is to determine the existence and number of the limit cycles of the polynomial differential system. In the general case, this is a very difficult task. Therefore, the researchers consider the weak Hilbert 16th problem. In addition, the existence of invariant algebraic curves in polynomial systems may influence the number of limit cycles. For example, the planar quadratic systems with one invariant line or conic curve or cubic curve can have at most one limit cycle [1-4]. In [3], the authors proved that the cubic systems with four invariant lines have at most one limit cycle. In $[5,6]$, the authors proved that a real polynomial system of degree $m$ with irreducible invariant algebraic curves has at most $1+(m-1)(m-2) / 2$ limit cycles if $m$ is even and $(m-1)(m-2) / 2$ limit cycles if $m$ is odd.

In this paper, we consider the weak Hilbert 16th problem that the unperturbed systems have a linear center and an invariant algebraic curve

$$
\begin{gathered}
\dot{x}=-y C(x, y)+\varepsilon P(x, y), \\
\dot{y}=x C(x, y)+\varepsilon Q(x, y),
\end{gathered}
$$

where $P(x, y)$ and $Q(x, y)$ are polynomials of degree $n$ in $\mathbb{R}^{2}$, the algebraic curve $C(x, y)$ satisfies $C(0,0) \neq 0$, and $\varepsilon \in \mathbb{R}$ is a sufficient small parameter.
It is obvious that, on the region $\Omega=\{(x, y) \mid C(x, y) \neq 0\}$, the system (1) is equivalent to the following form:

$$
\begin{gathered}
\dot{x}=-y+\varepsilon \frac{P(x, y)}{C(x, y)}, \\
\dot{y}=x+\varepsilon \frac{Q(x, y)}{C(x, y)} .
\end{gathered}
$$

When $\varepsilon=0$, system (2) is a Hamilton system with a family of ovals

$$
\gamma_{h}=\left\{(x, y) \in \mathbb{R}^{2} \mid H(x, y)=x^{2}+y^{2}=h, h>0\right\} .
$$

Define the Abelian integral

$$
\Phi(h)=\oint_{\gamma_{h}} \frac{P(x, y) \mathrm{d} y-Q(x, y) \mathrm{d} x}{C(x, y)},
$$

which is also called first-order Melnikov function of (2). According to the Poincaré-Pontryagin theorem [7], the number of isolated real zeros of $\Phi(h)$ controls the number of limit cycles of system (1) that bifurcate from the periodic annulus of the perturbed system (1) with $\varepsilon=0$. That is to say, when the $\Phi(h)$ does not vanish exactly, the maximum number of the isolated real zeros of $\Phi(h)$ is corresponding to the upper bound of the number of limit cycles which bifurcate from periodic annulus of unperturbed systems.

For the arbitrary polynomials $P(x, y), Q(x, y)$ of given degree $n$, the number of limit cycles of (2) depends on 
the different choices of $C(x, y)$. At present, several works have figured out this problem for the particular choices of $C(x, y)$. In [8], the authors studied the system (1) with $C(x, y)=1+x$ and proved that the number of limit cycles that bifurcate from the period orbits is at most $n$. The authors in $[9,10]$ studied the number of limit cycles which bifurcate from (1) when $\varepsilon=0$ with $C(x, y)=1+B x+A x^{2}$ and $C(x, y)=$ $1+a x+b y+c x\left(x^{2}+y^{2}\right)$, respectively. The authors in [11] studied the number of limit cycles of system (1) with $C(x, y)=$ $y^{2}+A x^{2}+B x+C$. In [12], the authors studied the number of limit cycles of system (1) with $C(x, y)=(x+a)(x+b)$ and obtain that the system can have at most $3[(n-1) / 2]+2$ limit cycles if $a \neq b$ and $2[(n-1) / 2]+1$ if $a=b$, respectively. In [13], the authors studied the case the curves $C(x, y)=0$ are three lines, two of them parallel and one perpendicular, and $[14,15]$ studied the case the curves are $k(k>3)$ lines, and any two of them are parallel or perpendicular directions. The authors in [16] studied the case the curves are consistent by $k$ nonzero points. The authors in [17] considered system (1) with $C(x, y)=1+x^{4}$ and proved that $3[(n+1) / 2]-2$ limit cycles can at most bifurcate from the periodic orbits of the unperturbed system. In [18], the authors proved that the system (1) with $C(x, y)=(1-x)^{m}$ has at most $n+m-1$ limit cycles.

The aim of this paper is to investigate the upper bound of the number of limit cycles bifurcate from the periodic annulus of the center of the unperturbed system (1) $(\varepsilon=$ 0 ) with the perturbed polynomials $P(x, y), Q(x, y)$ of given degree $n$, and $C(x, y)=1-x^{3}$ or $C(x, y)=1-x^{4}$.

Consider the planar differential system

$$
\begin{gathered}
\dot{x}=-y C(x, y)+\varepsilon \sum_{0 \leq k+j \leq n} a_{k, j} x^{k} y^{j}, \\
\dot{y}=x C(x, y)+\varepsilon \sum_{0 \leq k+j \leq n} b_{k, j} x^{k} y^{j},
\end{gathered}
$$

where $\varepsilon \in \mathbb{R}$ is a sufficient small parameter. Applying the Abelian integral, we obtain the following two main theorems.

Theorem 1. If $C(x, y)=1-x^{3}$, the lower bound of the maximum number of limit cycles bifurcating from the period orbits of system (5) with $\varepsilon=0$ is $4[(n+1) / 2]-2$.

Theorem 2. If $C(x, y)=1-x^{4}$, the upper bound of the maximum number of limit cycles bifurcating from the period orbits of system (5) with $\varepsilon=0$ is $3[(n+1) / 2]-2$.

Our primary purpose is to calculate the concrete expression of $\Phi(h)$; then we can obtain the number of limit cycles of the perturbed system (5) by determining the isolated real zeros of Abelian integral $\Phi(h)$. In Sections 2 and 3, we prove these two theorems with the different methods, respectively.

\section{The proof of Theorem 1}

Taking the change of variable $x=\sqrt{h} \cos \theta, y=\sqrt{h} \sin \theta(0<$ $h<1$ ), we have

$\Phi(h)$

$$
\begin{aligned}
& =\oint_{\gamma_{h}} \frac{\sum_{0 \leq k+j \leq n} a_{k, j} x^{k+1} y^{j}+\sum_{0 \leq k+j \leq n} b_{k, j} x^{k} y^{j+1}}{1-x^{3}} \mathrm{~d} \theta \\
& =\sum_{0 \leq k+j \leq n} h^{(k+j+1) / 2} \int_{0}^{2 \pi} \frac{a_{k, j} \cos ^{k+1} \theta \sin ^{j} \theta+b_{k, j} \cos ^{k} \theta \sin ^{j+1} \theta}{1-h^{3 / 2} \cos ^{3} \theta} \mathrm{d} \theta \\
& =\sum_{0 \leq k+j \leq n}\left(a_{k, j} \Phi_{k+1, j}(h)+b_{k, j} \Phi_{k, j+1}(h)\right),
\end{aligned}
$$

where

$$
\Phi_{k, j}=h^{(k+j) / 2} \int_{0}^{2 \pi} \frac{\cos ^{k} \theta \sin ^{j} \theta}{1-h^{3 / 2} \cos ^{3} \theta} \mathrm{d} \theta .
$$

Firstly, we have the following obvious result.

Lemma 3. If $j$ is odd, $\Phi_{k, j}=0(k \geq 0)$.

According to Lemma 3, we can rewrite the $\Phi(h)$ as follows:

$$
\begin{aligned}
& \Phi(h) \\
& =\sum_{s=1}^{n} a_{s, 0} \Phi_{s+1,0}+\sum_{s=1}^{n} \sum_{k+j=s}\left(a_{k, j}+b_{k+1, j-1}\right) \Phi_{k+1, j} \\
& \quad+\sum_{\substack{s=0 \\
s \text { odd }}}^{n} b_{0, s} \Phi_{0, s+1} .
\end{aligned}
$$

Denote $l=[(n+1) / 2]$ and $a_{k, j}^{*}=a_{k, j}+b_{k+1, j-1}(j \geq 1)$; then

$$
\begin{gathered}
\sum_{s=0}^{n} a_{s, 0} \Phi_{s+1,0}=\sum_{s=1}^{l}\left(a_{2 s-1,0} \Phi_{2 s, 0}+a_{2 s-2,0} \Phi_{2 s-1,0}\right) \\
\sum_{\substack{s=0 \\
s \text { odd }}}^{n} b_{0, s} \Phi_{0, s+1}=\sum_{s=1}^{l} b_{0,2 s-1} \Phi_{0,2 s} \\
\sum_{s=0}^{n} \sum_{k+j=s}\left(a_{k, j}+b_{k+1, j-1}\right) \Phi_{k+1, j} \\
=\sum_{s=1}^{l}\left(\sum_{m=1}^{s-1} a_{2 s-2 m-1,2 m}^{*} \Phi_{2 s-2 m, 2 m}\right. \\
\left.+\sum_{m=1}^{s-1} a_{2 s-2 m-2,2 m}^{*} \Phi_{2 s-2 m-1,2 m}\right) .
\end{gathered}
$$


Therefore, we have

$$
\begin{gathered}
\Phi(h) \\
=\sum_{s=1}^{l}\left(a_{2 s-1,0} \Phi_{2 s, 0}+\sum_{m=1}^{s-1} a_{2 s-2 m-1,2 m}^{*} \Phi_{2 s-2 m, 2 m}\right. \\
+b_{0,2 s-1} \Phi_{0,2 s}+a_{2 s-2,0} \Phi_{2 s-1,0} \\
\left.+\sum_{m=1}^{s-1} a_{2 s-2 m-2,2 m}^{*} \Phi_{2 s-2 m-1,2 m}\right) .
\end{gathered}
$$

Let

$$
\begin{gathered}
\Gamma_{0}=2 \pi, \quad \Gamma_{2 i}=\int_{0}^{2 \pi} \cos ^{2 i} \theta \mathrm{d} \theta=2 \pi \frac{(2 i-1) ! !}{2 i ! !} \quad(i \geq 1), \\
A_{k, j}=\sum_{r=0}^{k}(-1)^{r} C_{k}^{r} \Gamma_{2 j+2 r}
\end{gathered}
$$$$
B_{i, j}=a_{2 i-1,0} \Gamma_{2 i+6 j}
$$$$
+\sum_{m=1}^{i-1} a_{2 i-2 m-1,2 m}^{*} A_{m, i-m+3 j}+b_{0,2 i-1} A_{i, 3 j},
$$$$
C_{i, j}=a_{2 i-2,0} \Gamma_{2 i+2+6 j}+\sum_{m=1}^{i-1} a_{2 i-2 m-2,2 m}^{*} A_{m, i-m+3 j+1},
$$

where $a_{i, j}^{*}=0$ if $i<0$. Then, we have the following lemma.

Lemma 4. Abelian integral $\Phi(h)$ has an expansion in the form

$$
\begin{aligned}
& \Phi(h)=h\left(p_{0}+p_{1} h+p_{2} h^{2}+\cdots+p_{3 l} h^{3 l}\right. \\
&\left.+p_{3 l+1} h^{3 l+1}+p_{3 l+2} h^{3 l+2}+o\left(h^{3 l+2}\right)\right), \\
& h \in(0,1),
\end{aligned}
$$

where

$$
\begin{gathered}
p_{j}=\sum_{s=0}^{[(j+1) / 3]}\left(B_{j-3 s+1, s}+C_{j-3 s, s}\right), \quad \text { if } j<l, \\
p_{j}=\sum_{s=0}^{[l / 3]}\left(B_{l-3 s, u+s}+C_{l-3 s-1, u+s}\right), \\
\text { if } j=l+3 u-1 \quad(u=1,2,3, \ldots), \\
p_{j}=\sum_{s=0}^{[(l-2) / 3]} B_{l-3 s-2, u+s+1}+\sum_{s=0}^{[l / 3]} C_{j-3 s, u+s}, \\
p_{j}=\sum_{s=0}^{[(l-1) / 3]}\left(B_{l-3 s-1, u+s}+C_{l-3 s-2, u+s}\right), \\
\text { if } j=l+3 u-2 \quad(u=1,2,3, \ldots) .
\end{gathered}
$$

Proof. Firstly, we have

$$
\begin{aligned}
& \Phi_{2 s, 0} \\
& =h^{s} \int_{0}^{2 \pi} \frac{\cos ^{2 s} \theta}{1-h^{3 / 2} \cos ^{3} \theta} \mathrm{d} \theta \\
& =h^{s} \int_{0}^{2 \pi}\left(\cos ^{2 s} \theta+h^{3} \cos ^{2 s+6} \theta+\cdots\right. \\
& \left.+h^{3 i} \cos ^{2 s+6 i} \theta+\cdots\right) \mathrm{d} \theta \\
& =h^{s}\left(\Gamma_{2 s}+h^{3} \Gamma_{2 s+6}+\cdots+h^{3 i} \Gamma_{2 s+6 i}+\cdots\right) \text {, } \\
& \Phi_{2 s-2 m, 2 m} \\
& =h^{s} \int_{0}^{2 \pi} \frac{\cos ^{2 s-2 m} \theta \sin ^{2 m} \theta}{1-h^{3 / 2} \cos ^{3} \theta} \mathrm{d} \theta \\
& =h^{s} \sum_{r=0}^{m}(-1)^{r} C_{m}^{r} \int_{0}^{2 \pi}\left(\cos ^{2 s-2 m+2 r} \theta\right. \\
& +h^{3} \cos ^{2 s-2 m+6+2 r} \theta \\
& +\cdots+h^{3 i} \cos ^{2 s-2 m+2 r+6 i} \theta \\
& +\cdots) \mathrm{d} \theta \\
& =h^{s}\left(A_{m, s-m}+h^{3} A_{m, s-m+3}\right. \\
& \left.+\cdots+h^{3 i} A_{m, s-m+3 i}+\cdots\right), \\
& \Phi_{0,2 s} \\
& =h^{s} \int_{0}^{2 \pi} \frac{\sin ^{2 s} \theta}{1-h^{3 / 2} \cos ^{3} \theta} \mathrm{d} \theta \\
& =h^{s} \sum_{r=0}^{s}(-1)^{r} C_{s}^{r} \int_{0}^{2 \pi}\left(\cos ^{2 r} \theta+h^{3} \cos ^{2 r+6} \theta\right. \\
& +\cdots+h^{3 i} \cos ^{2 r+6 i} \theta \\
& +\cdots) \mathrm{d} \theta \\
& =h^{s}\left(A_{s, 0}+h^{3} A_{s, 3}+\cdots+h^{6} A_{s, 3 i}+\cdots\right), \\
& \Phi_{2 s-1,0} \\
& =h^{s-1 / 2} \int_{0}^{2 \pi} \frac{\cos ^{2 s-1} \theta}{1-h^{3 / 2} \cos ^{3} \theta} \mathrm{d} \theta \\
& =h^{s-1 / 2} \int_{0}^{2 \pi}\left(h^{3 / 2} \cos ^{2 s+2} \theta+h^{9 / 2} \cos ^{2 s+8} \theta+\cdots\right. \\
& \left.+h^{3 / 2+3 i} \cos ^{2 s+2+6 i} \theta \cdots\right) \mathrm{d} \theta \\
& =h^{s} \int_{0}^{2 \pi}\left(h \cos ^{2 s+2} \theta+h^{4} \cos ^{2 s+8} \theta\right. \\
& \left.+\cdots+h^{1+3 i} \cos ^{2 s+2+6 i} \theta \cdots\right) \mathrm{d} \theta \\
& =h^{s}\left(h \Gamma_{2 s+2}+h^{4} \Gamma_{2 s+8}+\cdots+h^{1+3 i} \Gamma_{2 s+2+6 i}+\cdots\right) \text {, }
\end{aligned}
$$




$$
\begin{aligned}
& \Phi_{2 s-2 m-1,2 m} \\
& =h^{s-1 / 2} \int_{0}^{2 \pi} \frac{\cos ^{2 s-2 m-1} \theta \sin ^{2 m} \theta}{1-h^{3 / 2} \cos ^{3} \theta} \mathrm{d} \theta \\
& =h^{s-1 / 2} \\
& \quad \times \sum_{r=0}^{m}(-1)^{r} C_{m}^{r} \int_{0}^{2 \pi}\left(h^{3 / 2} \cos ^{2 s-2 m+2+2 r} \theta\right. \\
& \quad+h^{9 / 2} \cos ^{2 s-2 m+8+2 r} \theta+\cdots \\
& \left.\quad+h^{3 / 2+3 i} \cos ^{2 s-2 m+2+2 r+6 i}+\cdots\right) \mathrm{d} \theta \\
& =h^{s}\left(h A_{m, s-m+1}+h^{4} A_{m, s-m+4}\right. \\
& \left.\quad+\cdots+h^{1+3 i} A_{m, s-m+1+3 i}+\cdots\right) .
\end{aligned}
$$

Substituting the previous formulas into (10), we have $\Phi(h)$

$=\sum_{s=1}^{l} h^{s}$

$\times\left[\left(a_{2 s-1,0} \Gamma_{2 s}+\sum_{m=1}^{s-1} a_{2 s-2 m-1,2 m}^{*} A_{m, s-m}\right.\right.$

$\left.+b_{0,2 s-1} A_{s, 0}\right)$

$+h^{3}\left(a_{2 s-1,0} \Gamma_{2 s+6}+\sum_{m=1}^{s-1} a_{2 s-2 m-1,2 m}^{*} A_{m, s-m+3}\right.$ $\left.+b_{0,2 s-1} A_{s, 3}\right)+\cdots$

$+h^{3 i}\left(a_{2 s-1,0} \Gamma_{2 s+6 i}+\sum_{m=1}^{s-1} a_{2 s-2 m-1,2 m}^{*} A_{m, s-m+3 i}\right.$ $\left.+b_{0,2 s-1} A_{s, 3 i}\right)+\cdots$

$+h\left(a_{2 s-2,0} \Gamma_{2 s+2}+\sum_{m=1}^{s-1} a_{2 s-2 m-2,2 m}^{*} A_{m, s-m+1}\right)$

$+h^{4}\left(a_{2 s-2,0} \Gamma_{2 s+8}+\sum_{m=1}^{s-1} a_{2 s-2 m-2,2 m}^{*} A_{m, s-m+4}\right)+\cdots$

$+h^{1+3 i}\left(a_{2 s-2,0} \Gamma_{2 s+6 i+2}\right.$

$\left.\left.+\sum_{m=1}^{s-1} a_{2 s-2 m-2,2 m}^{*} A_{m, s-m+3 i+1}\right)+\cdots\right]$.
Using (12) and (13), (17) can be written as follows:

$\Phi(h)$

$=\sum_{s=1}^{l} h^{s}\left(B_{s, 0}+h^{3} B_{s, 1}+\cdots+h^{3 i} B_{s, i}\right.$

$$
\left.+\cdots+h C_{s, 0}+h^{4} C_{s, 1}+\cdots+h^{1+3 i} C_{s, i}+\cdots\right)
$$

$=h\left[\left(B_{1,0}+h^{3} B_{1,1}+\cdots+h^{3 i} B_{1, i}+\cdots+h C_{1,0}\right.\right.$

$\left.+h^{4} C_{1,1}+\cdots+h^{1+3 i} C_{1, i}+\cdots\right)$

$+h\left(B_{2,0}+h^{3} B_{2,1}+\cdots+h^{3 i} B_{2, i}\right.$

$\left.+\cdots+h C_{2,0}+h^{4} C_{2,1}+\cdots+h^{1+3 i} C_{2, i}+\cdots\right)$

$+h^{2}\left(B_{3,0}+h^{3} B_{3,1}+\cdots+h^{3 i} B_{3, i}+\cdots+h C_{3,0}\right.$

$\left.+h^{4} C_{3,1}+\cdots+h^{1+3 i} C_{3, i}+\cdots\right)+\cdots$

$+h^{l-1}\left(B_{l, 0}+h^{3} B_{l, 1}+\cdots+h^{3 i} B_{l, i}+\cdots\right.$

$\left.\left.+h C_{l, 0}+h^{4} C_{l, 1}+\cdots+h^{1+3 i} C_{l, i}+\cdots\right)\right]$.

Thus, we have

if $j<l$,

$$
\begin{gathered}
p_{j}=B_{j+1,0}+B_{j-2,1}+\cdots+B_{j-3 i+1, i}+\cdots+C_{j, 0} \\
\quad+C_{j-3,1}+\cdots+C_{j-3 i, i}+\cdots, \\
\text { if } j=l+3 u-1(u=1,2,3, \ldots), \\
p_{j}=B_{l, u}+B_{l-3, u+1}+\cdots+B_{l-3 i, u+i} \\
+\cdots+C_{l-1, u}+C_{l-4, u+1}+\cdots+C_{l-3 i-1, u+i}+\cdots, \\
\text { if } j=l+3 u(u=0,1,2, \cdots), \\
p_{j}=B_{l-2, u+1}+B_{l-5, u+2}+\cdots+B_{l-3 i-2, u+i+1} \\
+\cdots+C_{l, u}+C_{l-3, u+1}+\cdots+C_{l-3 i, u+i}+\cdots \\
\quad \text { if } j=l+3 u-2(u=1,2,3, \ldots), \\
p_{j}=B_{l-1, u}+B_{l-4, u+2}+\cdots+B_{l-3 i-1, u+i}+\cdots \\
+C_{l-2, u}+C_{l-5, u+1}+\cdots+C_{l-3 i-2, u+i}+\cdots,
\end{gathered}
$$

where $i=0,1,2, \ldots$ and $B_{i, j}=C_{i, j}=0$ if $i<0$. The proof is completed. 
By Lemma 4 , we regard $a_{2 j-1,0}, a_{2 j-3,2}^{*}, \ldots, a_{1,2 j-2}^{*}, b_{0,2 j-1}$, $a_{2 j-2,0}, a_{2 j-4,2}^{*}, \ldots, a_{2,2 j-4}^{*}, a_{0,2 j-2}^{*}(j \geq 1)$ as free parameters, and denote vectors

$$
\begin{aligned}
& \alpha_{j}=\left(a_{2 j-1,0}, a_{2 j-3,2}^{*}, \ldots, a_{1,2 j-2}^{*}, b_{0,2 j-1}\right), \\
& \beta_{j}=\left(a_{2 j-2,0}, a_{2 j-4,2}^{*}, \ldots, a_{2,2 j-4}^{*}, a_{0,2 j-2}^{*}\right)
\end{aligned}
$$

and Jacobian matrices

$$
\begin{gathered}
J_{j}=\frac{\partial\left(p_{j-1}, p_{j+2}, p_{j+5}, \ldots, p_{4 j-1}\right)}{\partial \alpha_{j}}, \\
H_{j}=\frac{\partial\left(p_{j}, p_{j+3}, p_{j+6}, \ldots, p_{4 j-3}\right)}{\partial \beta_{j}}, \\
I_{l} \\
=\frac{\partial\left(p_{0}, p_{1}, p_{2}, \ldots, p_{l-2}, p_{l-1}, p_{l}, \ldots, p_{4 l-5}, p_{4 l-4}, p_{4 l-3}, p_{4 l-1}\right)}{\partial\left(\alpha_{1}, \beta_{1}, \alpha_{2}, \beta_{2}, \alpha_{3}, \beta_{3}, \ldots, \alpha_{l}, \beta_{l}\right)},
\end{gathered}
$$

where $j, l \geq 1$. Then, $J_{j}$ is $(j+2) \times(j+2)$ matrix, $H_{j}$ is $j \times j$ matrix, and $I_{l}$ is $(4 l-1) \times\left(l^{2}+2 l\right)$ matrix.

For matrices $J_{j}, H_{j}$ and $I_{l}$, we have Lemmas 5 and 6 , respectively.

Lemma 5. For $j \geq 1, \operatorname{det}\left(J_{j}\right) \neq 0$ and $\operatorname{det}\left(H_{j}\right) \neq 0$. That is, $\operatorname{rank}\left(J_{j}\right)=j+1, \operatorname{rank}\left(H_{j}\right)=j$.

Proof. According to Lemma 4 and (24), we can obtain that

$$
J_{j}=\left(\begin{array}{ccccccc}
\Gamma_{2 j} & A_{1, j-1} & A_{2, j-2} & \cdots & A_{j-2,2} & A_{j-1,1} & A_{j, 0} \\
\Gamma_{2 j+6} & A_{1, j+2} & A_{2, j+1} & \cdots & A_{j-2,5} & A_{j-1,4} & A_{j, 3} \\
\Gamma_{2 j+12} & A_{1, j+5} & A_{2, j+4} & \cdots & A_{j-2,8} & A_{j-1,7} & A_{j, 6} \\
\Gamma_{2 j+18} & A_{1, j+8} & A_{2, j+7} & \cdots & A_{j-2,11} & A_{j-1,10} & A_{j, 9} \\
\vdots & \vdots & \vdots & & \vdots & \vdots & \vdots \\
\Gamma_{8 j-6} & A_{1,4 j-4} & A_{2,4 j-5} & \cdots & A_{j-2,3 j-1} & A_{j-1,3 j-2} & A_{j, 3 j-3} \\
\Gamma_{8 j} & A_{1,4 j-1} & A_{2,4 j-2} & \cdots & A_{j-2,3 j+2} & A_{j-1,3 j+1} & A_{j, 3 j}
\end{array}\right) .
$$

Define $(j+1) \times(j+1)$ matrix

$$
\begin{aligned}
& \text { ith }
\end{aligned}
$$

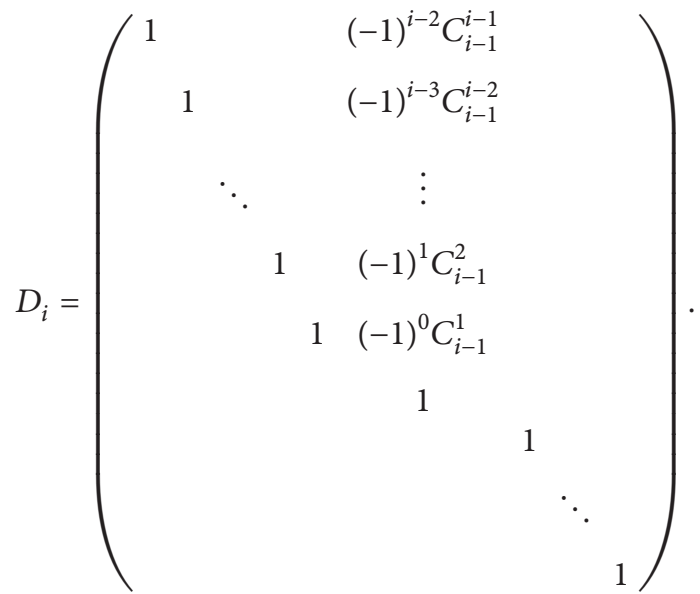

Then, we have

$$
J_{j}^{\prime} \equiv J_{j} D_{2} D_{3} \cdots D_{j+1}
$$

$$
=\left(\begin{array}{ccccccc}
\Gamma_{2 j} & \Gamma_{2 j-2} & \Gamma_{2 j-4} & \cdots & \Gamma_{4} & \Gamma_{2} & \Gamma_{0} \\
\Gamma_{2 j+6} & \Gamma_{2 j+4} & \Gamma_{2 j+2} & \cdots & \Gamma_{10} & \Gamma_{8} & \Gamma_{6} \\
\Gamma_{2 j+12} & \Gamma_{2 j+10} & \Gamma_{2 j+8} & \cdots & \Gamma_{16} & \Gamma_{14} & \Gamma_{12} \\
\Gamma_{2 j+18} & \Gamma_{2 j+16} & \Gamma_{2 j+14} & \cdots & \Gamma_{22} & \Gamma_{20} & \Gamma_{18} \\
\vdots & \vdots & \vdots & & \vdots & \vdots & \vdots \\
\Gamma_{8 j-6} & \Gamma_{8 j-8} & \Gamma_{8 j-10} & \cdots & \Gamma_{6 j-2} & \Gamma_{6 j-4} & \Gamma_{6 j-6} \\
\Gamma_{8 j} & \Gamma_{8 j-2} & \Gamma_{8 j-4} & \cdots & \Gamma_{6 j+4} & \Gamma_{6 j+2} & \Gamma_{6 j}
\end{array}\right) .
$$

Let $\Gamma_{i}^{j}=j ! ! / i ! ! ;$ then $\Gamma_{j}=2 \pi \Gamma_{j}^{j-1}(j \geq 1)$. Denote $T_{j-k}$

$$
=\left(\begin{array}{cccccc}
\Gamma_{2 j+6 k}^{2 j+4 k-1} & \Gamma_{2 j+6 k-2}^{2 j+4 k-3} & \cdots & \Gamma_{8 k+4}^{6 k+3} & \Gamma_{8 k+2}^{6 k+1} & \Gamma_{8 k}^{6 k-1} \\
\Gamma_{2 j+6 k+6}^{2 j+4 k+5} & \Gamma_{2 j+6 k+4}^{2 j+4 k+3} & \cdots & \Gamma_{8 k+10}^{6 k+9} & \Gamma_{8 k+8}^{6 k+7} & \Gamma_{8 k+6}^{6 k+5} \\
\vdots & \vdots & & \vdots & \vdots & \vdots \\
\Gamma_{8 j-6}^{8 j-2 k-7} & \Gamma_{8 j-8}^{8 j-2 k-9} & \cdots & \Gamma_{6 j+2 k-2}^{6 j-3} & \Gamma_{6 j+2 k-4}^{6 j-5} & \Gamma_{6 j+2 k-6}^{6 j-7} \\
\Gamma_{8 j}^{8 j-2 k-1} & \Gamma_{8 j-2}^{8 j-2 k-3} & \cdots & \Gamma_{6 j+2 k+4}^{6 j+3} & \Gamma_{6 j+2 k+2}^{6 j+1} & \Gamma_{6 j+2 k}^{6 j-1}
\end{array}\right)
$$

where $T_{j-k}$ is $(j-k+1) \times(j-k+1)$ matrix. Then, $\operatorname{det}\left(J_{j}\right)=$ $\operatorname{det}\left(J_{j}^{\prime}\right)=(2 \pi)^{j+1} \operatorname{det}\left(T_{j}\right)$. 
We add entries of the $i+1$ th $(0<i \leq j-k+1)$ column which times $-(2(j+2-i)-1) / 2(j+2-i)$ to the $i$ th column and obtain

$$
\begin{aligned}
& T_{j-k} \longrightarrow T_{j-k}^{1}
\end{aligned}
$$

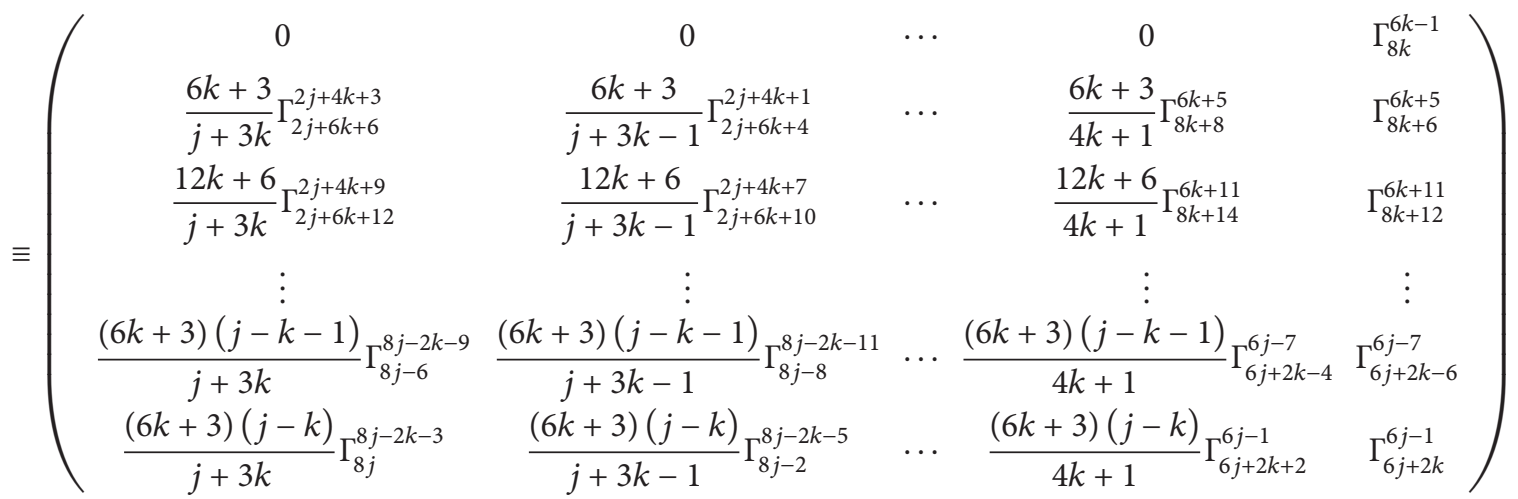

$$
\begin{aligned}
& \equiv\left(\begin{array}{cc}
\mathbf{0}_{1 \times(j-k)} & \Gamma_{8 k}^{6 k-1} \\
T_{j-k-1}^{0} & a_{k}
\end{array}\right) \text {, }
\end{aligned}
$$

where $a_{k}=\left(\Gamma_{8 k+6}^{6 k+5}, \Gamma_{8 k+12}^{6 k+11}, \ldots, \Gamma_{6 j+2 k-6}^{6 j-7}, \Gamma_{6 j+2 k}^{6 j-1}\right)^{T}$. We can write $T_{j-k-1}^{0}$ as follows:

$$
\begin{aligned}
& T_{j-k-1}^{0} \\
& =\left(\begin{array}{cccc}
6 k+3 & & & \\
& 12 k+6 & & \\
& & \ddots & \\
& & & (6 k+3)(j-k)
\end{array}\right) \\
& \times T_{j-k-1}\left(\begin{array}{cccc}
\frac{1}{j+3 k} & & & \\
& \frac{1}{j+3 k-1} & & \\
& & \ddots & \\
& & & \frac{1}{4 k+1}
\end{array}\right) \text {; }
\end{aligned}
$$

then $\operatorname{det}\left(T_{j-k}\right)=(-1)^{j-k+2} \Gamma_{8 k}^{6 k-1}(6 k+3)^{j-k}(j-k) !((4 k) ! /(j+$ $3 k)$ !) $\operatorname{det}\left(T_{j-k-1}\right)$.

Summarizing above results, we have

$$
\begin{aligned}
\operatorname{det}\left(J_{j}\right)= & (2 \pi)^{j+1}(-1)^{(j+2)+(j+1)+\cdots+3} 3^{j} 9^{j-1} 15^{j-2} \\
& \cdots(6 j-9)^{2}(6 j-3) j !(j-1) ! \cdots 2 ! \\
& \times \Gamma_{8}^{5} \Gamma_{16}^{11} \Gamma_{24}^{17} \cdots \Gamma_{8 j}^{6 j-1} \frac{1}{j !} \frac{4 !}{(j+3) !} \frac{8 !}{(j+6) !} \\
& \cdots \frac{(4 j-4) !}{(4 j-3) !} \neq 0
\end{aligned}
$$

therefore, $\operatorname{rank}\left(J_{j}\right)=j+1$.
According to Lemma 4 and (25), we have

$$
\begin{aligned}
& H_{j-1} \\
& =\left(\begin{array}{cccccc}
\Gamma_{2 j} & A_{1, j-1} & A_{2, j-2} & \cdots & A_{j-3,3} & A_{j-2,2} \\
\Gamma_{2 j+6} & A_{1, j+2} & A_{2, j+1} & \cdots & A_{j-3,6} & A_{j-2,5} \\
\Gamma_{2 j+12} & A_{1, j+5} & A_{2, j+4} & \cdots & A_{j-3,9} & A_{j-2,8} \\
\vdots & \vdots & \vdots & & \vdots & \vdots \\
\Gamma_{8 j-18} & A_{1,4 j-10} & A_{2,4 j-11} & \cdots & A_{j-3,3 j-6} & A_{j-2,3 j-7} \\
\Gamma_{8 j-12} & A_{1,4 j-7} & A_{2,4 j-8} & \cdots & A_{j-3,3 j-3} & A_{j-2,3 j-4}
\end{array}\right) .
\end{aligned}
$$

By the above proof procedure, we can obtain $\operatorname{rank}\left(H_{j-1}\right)=$ $j-1$ in a similar way. That is $\operatorname{rank}\left(H_{j}\right)=j$. The proof is completed.

Lemma 6. For $l \geq 1, \operatorname{rank}\left(I_{l}\right)=4 l-1$.

Proof. Firstly, if $l=1$,

$$
\begin{aligned}
I_{1} & =\frac{\partial\left(p_{0}, p_{1}, p_{3}\right)}{\partial\left(\alpha_{1}, \beta_{1}\right)} \\
& =\left(\begin{array}{ccc}
\Gamma_{2} & A_{1,0} & 0 \\
0 & 0 & \Gamma_{4} \\
\Gamma_{8} & A_{1,3} & 0
\end{array}\right) \longrightarrow\left(\begin{array}{ccc}
\Gamma_{2} & A_{1,0} & 0 \\
\Gamma_{8} & A_{1,3} & 0 \\
0 & 0 & \Gamma_{4}
\end{array}\right),
\end{aligned}
$$




$$
\begin{aligned}
& \text { If } l=2 \text {, we have } \\
& I_{2}=\frac{\partial\left(p_{0}, p_{1}, p_{2}, p_{3}, p_{4}, p_{5}, p_{7}\right)}{\partial\left(\alpha_{1}, \beta_{1}, \alpha_{2}, \beta_{2}\right)} \\
& =\left(\begin{array}{cccccccc}
\Gamma_{2} & A_{1,0} & 0 & 0 & 0 & 0 & 0 & 0 \\
0 & 0 & \Gamma_{4} & A_{1,1} & A_{2,0} & \Gamma_{4} & 0 & 0 \\
0 & 0 & 0 & 0 & 0 & 0 & \Gamma_{6} & A_{1,2} \\
\Gamma_{8} & A_{1,3} & 0 & 0 & 0 & 0 & 0 & 0 \\
0 & 0 & \Gamma_{10} & A_{1,4} & A_{2,3} & \Gamma_{10} & 0 & 0 \\
0 & 0 & 0 & 0 & 0 & 0 & \Gamma_{12} & A_{1,5} \\
0 & 0 & \Gamma_{16} & A_{1,7} & A_{2,6} & \Gamma_{16} & 0 & 0
\end{array}\right), \\
& \longrightarrow\left(\begin{array}{cccccccc}
\Gamma_{2} & A_{1,0} & 0 & 0 & 0 & 0 & 0 & 0 \\
\Gamma_{8} & A_{1,3} & 0 & 0 & 0 & 0 & 0 & 0 \\
0 & 0 & \Gamma_{4} & A_{1,1} & A_{2,0} & 0 & 0 & 0 \\
0 & 0 & \Gamma_{10} & A_{1,4} & A_{2,3} & 0 & 0 & 0 \\
0 & 0 & \Gamma_{16} & A_{1,7} & A_{2,6} & 0 & 0 & 0 \\
0 & 0 & 0 & 0 & 0 & 0 & \Gamma_{6} & A_{1,2} \\
0 & 0 & 0 & 0 & 0 & 0 & \Gamma_{12} & A_{1,5}
\end{array}\right) \\
& \longrightarrow\left(\begin{array}{llll}
J_{1} & & & \\
& J_{2} & & \\
& & H_{2} & \mathbf{0}_{2 \times 1}
\end{array}\right) .
\end{aligned}
$$

Then, by Lemma 5, $\operatorname{rank}\left(I_{2}\right)=\operatorname{rank}\left(J_{1}\right)+\operatorname{rank}\left(J_{2}\right)+$ $\operatorname{rank}\left(H_{2}\right)=7$.

For $l \geq 3$, let $\gamma_{l}^{1}=\left(p_{0}, p_{1}, \ldots, p_{l-3}\right), \gamma_{l}^{2}=\left(p_{l-2}, p_{l+1}\right.$, $\left.\ldots, p_{4 l-5}\right), \gamma_{l}^{3}=\left(p_{l-1}, p_{l+2}, \ldots, p_{4 l-1}\right), \gamma_{l}^{4}=\left(p_{l}, p_{l+3}, \ldots\right.$, $\left.p_{4 l-3}\right)$; we have

$$
I_{l} \longrightarrow I_{l}^{*}=\frac{\partial\left(\gamma_{l}^{1}, \gamma_{l}^{2}, \gamma_{l}^{3}, \gamma_{l}^{4}\right)}{\partial\left(\alpha_{1}, \beta_{1}, \alpha_{2}, \beta_{2}, \ldots, \alpha_{l}, \beta_{l}\right)} .
$$

According to Lemma 4 and the definitions of $J_{j}$ and $H_{j}$, simplifying it by elementary transformation of matrix, we obtain

$$
I_{l}^{*} \longrightarrow\left(\begin{array}{ccccc}
E_{l-2} & & & & \\
& J_{l-1} & & & \\
& & J_{l} & & \\
& & & H_{l} & \mathbf{0}_{l \times\left(l^{2}-2 l+1\right)}
\end{array}\right) .
$$

Therefore, $\operatorname{rank}\left(I_{l}\right)=\operatorname{rank}\left(E_{1-2}\right)+\operatorname{rank}\left(J_{l-1}\right)+\operatorname{rank}\left(J_{l}\right)+$ $\operatorname{rank}\left(H_{l}\right)=4 l-1$. The proof is completed.

By Lemma 6 , it is obvious that $p_{0}, p_{1}, p_{2}, \ldots, p_{l-2}$, $p_{l-1}, p_{l}, \ldots, p_{4 l-4}, p_{4 l-3}, p_{4 l-1}$ are independent. Now, we have the following lemma.

Lemma 7. For $l \geq 1$, one can write $p_{j}$ as follows:

$$
\begin{array}{r}
p_{l+3 u-1}=x_{1} p_{l-1}+x_{2} p_{l+2}+\cdots+x_{l+1} p_{4 l-1}, \\
u>l, \\
p_{l+3 u-2}=y_{1} p_{l-2}+y_{2} p_{l+1}+\cdots+y_{l} p_{4 l-5}, \\
u>l-1, \\
p_{l+3 u}=z_{1} p_{l+1}+z_{2} p_{l+4}+\cdots+z_{l} p_{4 l-3}, \\
u>l-1,
\end{array}
$$

where $\left(x_{1}, x_{2}, \ldots, x_{l+1}\right),\left(y_{1}, y_{2}, \ldots, y_{l}\right)$ and $\left(z_{1}, z_{2}, \ldots, z_{l}\right)$ are nonzero vectors.

Proof. According to Lemma 4 , if $j=l+3 u-1(u=1,2,3, \ldots)$,

$$
p_{j}=\sum_{s=0}^{[l / 3]}\left(B_{l-3 s, u+s}+C_{l-3 s-1, u+s}\right) .
$$

Substituting $p_{l-1}, p_{l+2}, \ldots, p_{4 l-1}$ into the first equation, we obtain a linear equation

$$
d_{l+3 u-1}=J_{l}^{T} X \quad(u>l)
$$

where $X=\left(x_{1}, x_{2}, \ldots, x_{l+1}\right)^{T}, d_{l+3 u-1}=\left(\Gamma_{2 l+6 u}, A_{1, l+3 u}\right.$, $\left.A_{2, l+3 u-2}, \ldots, A_{l-1,3 u+1}, A_{l, 3 u}\right)^{T}$. According to Lemma 5, $\operatorname{det}\left(J_{l}^{T}\right) \neq 0$, (41) has unique solution $X=\left(x_{1}^{\prime}, x_{2}^{\prime}, \ldots\right.$, $\left.x_{l+1}^{\prime}\right)^{T} \neq \mathbf{0}$. That is, the first formula holds.

If $j=l+3 u-2(u=1,2,3, \ldots)$, in a similar way, we can prove that the second formula holds.

If $j=l+3 u(u=0,1,2, \ldots)$, we have

$$
p_{j}=\sum_{s=0}^{[(l-2) / 3]} B_{l-3 s-2, u+s+1}+\sum_{s=0}^{[l / 3]} C_{j-3 s, u+s},
$$

substituting $p_{l+1}, p_{l+4}, \ldots, p_{4 l-3}$ into the third equation, we obtain a linear equation

$$
c_{l+3 u}=H_{l}^{T} Z \quad(u>l-1),
$$

where $Z=\left(z_{1}, z_{2}, \ldots, z_{l}\right)^{T}, c_{l+3 u}=\left(\Gamma_{2 l+6 u+2}, A_{1, l+3 u}, A_{2, l+3 u-1}\right.$, $\left.\ldots, A_{l-2,3 u+3}, A_{l-1,3 u+2}\right)^{T}$. According to Lemma 5, $\operatorname{det}\left(H_{l}^{T}\right) \neq 0$, (43) has unique solution $Z=\left(z_{1}^{\prime}, z_{2}^{\prime}, \ldots\right.$, $\left.z_{l}^{\prime}\right)^{T} \neq \mathbf{0}$. That is, the third formula also holds. The proof is completed.

Now, we prove Theorem 1.

Proof. For $h \in(0,1)$, Abelian integral $\Phi(h)$ has an expansion of the following form:

$$
\begin{aligned}
\Phi(h)=h\left(p_{0}+p_{1} h+p_{2} h^{2}+\cdots+p_{4 l-3} h^{4 l-3}\right. \\
\left.+p_{4 l-2} h^{4 l-2}+p_{4 l-1} h^{4 l-1}+o\left(h^{4 l-1}\right)\right) .
\end{aligned}
$$

According to Lemma 6, for $l \geq 1, p_{0}, p_{1}, p_{2}, \ldots, p_{l-2}, p_{l-1}$, $p_{l}, \ldots, p_{4 l-4}, p_{4 l-3}, p_{4 l-1}$ are independent.

Let $p_{0}=p_{1}=p_{2}=\cdots=p_{4 l-1}=p_{4 l-3}=0$ and $p_{4 u-1}=$ 1 ; then, by Lemma 7, $p_{4 l-2}=0$. Thus (44) becomes $\Phi(h)=$ $h^{4 l}+o\left(h^{4 l}\right)$, and $\Phi(h)>0$ if $h \in(0,1)$. Furthermore, we take $p_{0}=p_{1}=p_{2}=\cdots=p_{4 l-5}=p_{4 l-4}=0$, then $p_{4 l-2}=0$ still holds. Choosing proper $p_{4 l-3} \in(-1,0)$ such that $\Phi(h)=$ $p_{4 l-3} h^{4 l-2}+h^{4 l}+o\left(h^{4 l}\right)<0$, by Descartes' rule of signs, (44) has a root $h_{1}$ on interval $(0,1)$.

Let $p_{0}=p_{1}=p_{2}=\cdots=p_{4 l-6}=p_{4 l-5}=0$, and choose proper $p_{4 l-4}\left(p_{4 l-4} \in(0,1)\right)$ so that $\Phi(h)=$ $p_{4 l-4} h^{4 l-3}+p_{4 l-3} h^{4 l-2}+h^{4 l}+o\left(h^{4 l}\right)>0$; then (44) has the second root $h_{2}$ on interval $(0,1)$. In a similar way, we take proper $p_{i}(i=4 l-6,4 l-7, \ldots, 2,1,0)$ in turn such that 
$p_{i} p_{i-1}<0$ and $\left|p_{i}\right| \in(0,1)$. According to Descartes' rule of signs, we can obtain $4 l-4$ zeros $h_{3}, h_{4}, \ldots, h_{4 l-3}, h_{4 l-2}$ on interval $(0,1)$.

Applying the Poincaré-Pontryagin theorem, the system (5) with $C(x, y)=1-x^{3}$ can have at least $4[(n+1) / 2]-2$ limit cycles for suitable $a_{k, j}$ and $b_{k, j}(0 \leq k+j \leq n)$. The proof of Theorem 1 is completed.

\section{The proof of Theorem 2}

In this section, we will prove Theorem 2. At first, all the primary computations to express the Abelian integral $\Phi(h)$ and some concerned lemmas are presented.

Taking the change of variable $x=\sqrt{h} \cos \theta, y=\sqrt{h} \sin \theta$ $(0<h<1)$, then by $(4)$ we have

$$
\begin{aligned}
\Phi(h) & =\oint_{\gamma_{h}} \frac{\sum_{0 \leq k+j \leq n} a_{k, j} x^{k+1} y^{j}+\sum_{0 \leq k+j \leq n} b_{k, j} x^{k} y^{j+1}}{1-x^{4}} \mathrm{~d} \theta \\
& =\frac{1}{2}\left(\Phi^{1}+\Phi^{2}\right),
\end{aligned}
$$

where

$$
\begin{aligned}
& \Phi^{1}=\oint_{\gamma_{h}} \frac{\sum_{0 \leq k+j \leq n} a_{k, j} x^{k+1} y^{j}+\sum_{0 \leq k+j \leq n} b_{k, j} x^{k} y^{j+1}}{1-x^{2}} \mathrm{~d} \theta, \\
& \Phi^{2}=\oint_{\gamma_{h}} \frac{\sum_{0 \leq k+j \leq n} a_{k, j} x^{k+1} y^{j}+\sum_{0 \leq k+j \leq n} b_{k, j} x^{k} y^{j+1}}{1+x^{2}} \mathrm{~d} \theta .
\end{aligned}
$$

Denote

$$
\begin{aligned}
\Phi_{k, j}^{1} & =\oint_{\gamma_{h}} \frac{x^{k} y^{j}}{1-x^{2}} \mathrm{~d} \theta=\oint_{\gamma_{h}} \frac{x^{k} y^{j}}{(1-x)(1+x)} \mathrm{d} \theta, \\
\Phi_{k, j}^{2} & =\oint_{\gamma_{h}} \frac{x^{k} y^{j}}{1+x^{2}} \mathrm{~d} \theta=\oint_{\gamma_{h}} \frac{x^{k} y^{j}}{(1-i x)(1+i x)} \mathrm{d} \theta,
\end{aligned}
$$

where $i^{2}=-1$; then we have

$$
\begin{aligned}
& \Phi^{1}=\sum_{0 \leq k+j \leq n}\left(a_{k, j} \Phi_{k+1, j}^{1}+b_{k, j} \Phi_{k, j+1}^{1}\right), \\
& \Phi^{2}=\sum_{0 \leq k+j \leq n}\left(a_{k, j} \Phi_{k+1, j}^{2}+b_{k, j} \Phi_{k, j+1}^{2}\right) .
\end{aligned}
$$

Lemma 8. Let $\omega_{1}=1, \omega_{2}=-1, \omega_{3}=i, \omega_{4}=-i$; then

$$
\Psi_{s}=\oint_{\gamma_{h}} \frac{\mathrm{d} \theta}{1-\omega_{s} x}=\frac{2 \pi}{\sqrt{1-\omega_{s}^{2} h}},
$$

where $s=1,2,3,4$. And

$$
\begin{gathered}
\Phi_{0,0}^{1}=\oint_{\gamma_{h}} \frac{\mathrm{d} \theta}{(1-x)(1+x)}=\frac{2 \pi}{\sqrt{1-h}}, \\
\Phi_{1,0}^{1}=\oint_{\gamma_{h}} \frac{x \mathrm{~d} \theta}{(1-x)(1+x)}=0, \\
\Phi_{0,0}^{2}=\oint_{\gamma_{h}} \frac{\mathrm{d} \theta}{(1-i x)(1+i x)}=\frac{2 \pi}{\sqrt{1+h}}, \\
\Phi_{1,0}^{2}=\oint_{\gamma_{h}} \frac{x \mathrm{~d} \theta}{(1-i x)(1+i x)}=0 .
\end{gathered}
$$

Proof. We use the residue theorem to compute the $\Psi_{s}(s=$ $1,2,3,4)$.

When $x=\sqrt{h} \cos \theta$, we have

$$
\Psi_{s}=\oint_{\gamma_{h}} \frac{\mathrm{d} \theta}{1-\omega_{s} x}=\int_{0}^{2 \pi} \frac{\mathrm{d} \theta}{1-\omega_{s} \sqrt{h} \cos \theta} .
$$

Let $e^{i \theta}=z$; then $\cos \theta=\left(z^{2}+1\right) / 2 z, \mathrm{~d} \theta=\mathrm{d} z / i z$. The previous formula becomes

$$
\begin{aligned}
\Psi_{s} & =\oint_{|z|=1} \frac{1}{1-\omega_{s} \sqrt{h} \cdot\left(\left(z^{2}+1\right) / 2 z\right)} \frac{\mathrm{d} z}{i z} \\
& =-\frac{2}{i} \oint_{|z|=1} \frac{\mathrm{d} z}{\omega_{s} \sqrt{h} z^{2}-2 z+\omega_{s} \sqrt{h}} \\
& =-4 \pi \operatorname{Res}\left[\frac{1}{\omega_{s} \sqrt{h} z^{2}-2 z+\omega_{s} \sqrt{h}}, z_{1}=\frac{1-\sqrt{1-\omega_{s}^{2} h}}{\omega_{s} \sqrt{h}}\right] \\
& =-4 \pi \lim _{z \rightarrow z_{1}} \frac{z-z_{1}}{\omega_{s} \sqrt{h} z^{2}-2 z+\omega_{s} \sqrt{h}} \\
& =\frac{2 \pi}{\sqrt{1-\omega_{s}^{2} h}} ;
\end{aligned}
$$

hence (49) holds. For the first formula of (50),

$$
\begin{aligned}
\Phi_{0,0}^{1} & =\frac{1}{2}\left(\oint_{\gamma_{h}} \frac{\mathrm{d} \theta}{1-\omega_{1} x}+\oint_{\gamma_{h}} \frac{\mathrm{d} \theta}{1+\omega_{2} x}\right) \\
& =\frac{1}{2}\left(\frac{2 \pi}{\sqrt{1-h}}+\frac{2 \pi}{\sqrt{1-h}}\right)=\frac{2 \pi}{\sqrt{1-h}},
\end{aligned}
$$

and the others can be proved in a similar way. The proof is completed.

Lemma 9. If $j$ is odd, the integrands in $\Phi_{k, j}^{1}$ and $\Phi_{k, j}^{2}$ are odd functions with respect to the variable $\theta$; therefore, $\Phi_{k, j}^{1}=0$ and $\Phi_{k, j}^{2}=0$.

Define

$$
\Phi_{k, j}=\oint_{\gamma_{h}} \frac{a_{k, j} x^{k+1} y^{j}+b_{k, j} x^{k} y^{j+1}}{1-x^{4}} \mathrm{~d} \theta
$$

then, according to Lemmas 8 and 9 and the definition of $\Phi(h)$, one knows that $\Phi_{0,0}=0$. 
Lemma 10. If $j$ is even, then

$$
\begin{aligned}
& \Phi_{k, j}^{1}=\sum_{s=0}^{j / 2}(-1)^{s} h^{j /(2-s)} C_{j / 2}^{s} \Phi_{k+2 s, 0}^{1}, \\
& \Phi_{k, j}^{2}=\sum_{s=0}^{j / 2}(-1)^{s} h^{j /(2-s)} C_{j / 2}^{s} \Phi_{k+2 s, 0}^{2} .
\end{aligned}
$$

Proof. If $j$ is even, then

$$
\begin{aligned}
\Phi_{k, j}^{1} & =\oint_{\gamma_{h}} \frac{x^{k} y^{j}}{1-x^{2}} \mathrm{~d} \theta=\oint_{\gamma_{h}} \frac{x^{k}\left(h-x^{2}\right)^{j / 2}}{1-x^{2}} \mathrm{~d} \theta \\
& =\oint_{\gamma_{h}} \sum_{s=0}^{j / 2}(-1)^{s} h^{j / 2-s} C_{j / 2}^{s} \frac{x^{k+2 s}}{1-x^{2}} \mathrm{~d} \theta \\
& =\sum_{s=0}^{j / 2}(-1)^{s} h^{j / 2-s} C_{j / 2}^{s} \Phi_{k+2 s, 0}^{1} .
\end{aligned}
$$

Similarly, (56) also holds. The proof is completed.

Lemma 11. (i) If $k$ is odd, $\Phi_{k, 0}^{1}=0$.

(ii) If $k$ is even, then

$$
\begin{aligned}
\Phi_{k, 0}^{1}= & \frac{2 \pi}{\sqrt{1-h}} \\
& \times\left[1-\sum_{\substack{l=1 \\
l \equiv k(\bmod 2)}}^{k} 2^{l-k} h^{(k-l) / 2} \sqrt{1-h} C_{k-l}^{(k-l) / 2}\right] .
\end{aligned}
$$

Proof. When $k>1$,

$$
\begin{aligned}
\Phi_{k, 0}^{1} & =\oint_{\gamma_{h}} \frac{x^{k}}{1-x^{2}} \mathrm{~d} \theta=\frac{1}{2}\left(\oint_{\gamma_{h}} \frac{x^{k}}{1-x} \mathrm{~d} \theta+\oint_{\gamma_{h}} \frac{x^{k}}{1+x} \mathrm{~d} \theta\right) \\
& \equiv \frac{1}{2}\left(M_{1}+M_{2}\right) .
\end{aligned}
$$

We use the residue theorem to compute the integrals $M_{1}$ and $M_{2}$. Denote $e^{i \theta}=z$; thus, $\cos \theta=\left(z^{2}+1\right) / 2 z, \mathrm{~d} \theta=\mathrm{d} z / i z$. We have

$M_{1}$

$$
\begin{aligned}
& =\oint_{\gamma_{h}} \frac{x^{k}}{1-x} \mathrm{~d} \theta=\oint_{|z|=1} \frac{\left(\sqrt{h} \cdot\left(\left(z^{2}+1\right) / 2 z\right)\right)^{k}}{1-\sqrt{h} \cdot\left(\left(z^{2}+1\right) / 2 z\right)} \cdot \frac{1}{i z} \mathrm{~d} z \\
& =-\frac{2^{1-k} \cdot h^{k / 2}}{i} \oint_{\gamma_{h}} \frac{\left(z^{2}+1\right)^{k} \cdot\left(\sqrt{h} z^{2}-2 z+\sqrt{h}\right)}{\mathrm{d} z} \\
& =-2^{2-k} \pi h^{k / 2}\left[\operatorname{Re} s\left(M_{1}, 0\right)+\operatorname{Re} s\left(M_{1}, z_{1}=\frac{1-\sqrt{1-h}}{\sqrt{h}}\right)\right] .
\end{aligned}
$$

Since $z_{1}=(1-\sqrt{1-h}) / \sqrt{h}$ is the first-order zero of the equation $\sqrt{h} z^{2}-2 z+\sqrt{h}=0$, the residue of $M_{1}$ at $z_{1}$ is

$$
\begin{aligned}
\operatorname{Res}\left(M_{1}, z_{1}\right) & =\lim _{z \rightarrow z_{1}} \frac{\left(z^{2}+1\right)^{k} \cdot\left(\sqrt{h} z^{2}-2 z+\sqrt{h}\right)}{z^{k}} \cdot\left(z-z_{1}\right) \\
& =\frac{-2^{k-1}}{h^{k / 2} \cdot \sqrt{1-h}} .
\end{aligned}
$$

For the residue at $z=0$, we have the expansion of $M_{1}$ in the form

$$
\begin{aligned}
M_{1} & =\frac{\left(z^{2}+1\right)^{k}}{z^{k} \cdot\left(\sqrt{h} z^{2}-2 z+\sqrt{h}\right)} \\
& =\frac{1}{2 z} \cdot \frac{\left(\left(z^{2}+1\right) / z\right)^{k}}{(\sqrt{h} / 2) \cdot\left(\left(z^{2}+1\right) / z\right)-1} \\
& =\sum_{l=1}^{\infty} 2^{l-1} h^{-l / 2} z^{-1}\left(\frac{z^{2}+1}{z}\right)^{k-l} ;
\end{aligned}
$$

the coefficient of $z^{-1}$ is corresponding to the residue of $M_{1}$ at $z=0$; therefore,

$$
\operatorname{Re} s\left(M_{1}, 0\right)=\sum_{\substack{l=1 \\ l \equiv k(\bmod 2)}}^{k} 2^{l-1} h^{-l / 2} C_{k-l}^{(k-l) / 2} .
$$

Substituting them into (60), we have

$$
\begin{aligned}
& M_{1} \\
& =-2^{2-k} \pi h^{k / 2}\left[\frac{-2^{k-1}}{h^{k / 2} \cdot \sqrt{1-h}}+\sum_{\substack{l=1 \\
l \equiv k(\bmod 2)}}^{k} 2^{l-1} h^{-l / 2} C_{k-l}^{(k-l) / 2}\right] \\
& =\frac{2 \pi}{\sqrt{1-h}}\left[1-\sum_{\substack{l=1 \\
l \equiv k(\bmod 2)}}^{k} 2^{l-k} h^{(k-l) / 2} \sqrt{1-h} C_{k-l}^{(k-l) / 2}\right] .
\end{aligned}
$$

We can compute $M_{2}$ in a similar way and obtain

$$
\begin{aligned}
M_{2} & \frac{2 \pi}{\sqrt{1-h}} \\
& \times\left[(-1)^{k}+\sum_{\substack{l=1 \\
l=k(\bmod 2)}}^{k}(-1)^{l-1} 2^{l-k} h^{(k-l) / 2} \sqrt{1-h} C_{k-l}^{(k-l) / 2}\right] .
\end{aligned}
$$


By the formulas (64) and (65), $\Phi_{k, 0}^{1}$ becomes

$$
\begin{aligned}
\Phi_{k, 0}^{1}= & \frac{\pi}{\sqrt{1-h}} \\
\times & {\left[1-\sum_{\substack{l=1 \\
l \equiv k(\bmod 2)}}^{k} 2^{l-k} h^{(k-l) / 2} \sqrt{1-h} C_{k-l}^{(k-l) / 2}+(-1)^{k}\right.} \\
& \left.+\sum_{\substack{l=1 \\
l \equiv k(\bmod 2)}}^{k}(-1)^{l-1} 2^{l-k} h^{(k-l) / 2} \sqrt{1-h} C_{k-l}^{(k-l) / 2}\right],
\end{aligned}
$$

from the previous formula, it is easy to know that, if $k$ is odd, $\Phi_{k, 0}^{1}=0$, and if $k$ is even, formula (58) is obtained. The proof is completed.

In a similar way, we can prove the following lemma.

Lemma 12. (i) If $k$ is odd, $\Phi_{k, 0}^{2}=0$.

(ii) If $k$ is even, then

$$
\begin{aligned}
\Phi_{k, 0}^{2}= & \frac{2 \pi i^{k}}{\sqrt{1+h}} \\
& \times\left[1-\sum_{\substack{l=1 \\
l \equiv k(\bmod 2)}}^{k} 2^{l-k} h^{(k-l) / 2} i^{-k-l} \sqrt{1+h} C_{k-l}^{(k-l) / 2}\right] .
\end{aligned}
$$

Using Lemmas 11 and 12, it is easy to see that, if $k$ is even, $\Phi_{k, 0}=0$. Therefore, by Lemma 10 and the definition of $\Phi_{k, j}$, if $n$ is even, $\Sigma_{k+j=n} \Phi_{k, j}=0$.

Lemma 13. Consider a function of the form

$$
F(x)=P_{0}(x)+\frac{P_{1}(x)}{\sqrt{1+x}}+\frac{P_{2}(x)}{\sqrt{1-x}},
$$

where $P_{j}(x)(j=1,2)$ are real polynomials of degree $n$ and the degree of $P_{0}$ is $n_{0}$. Then the number $\mathscr{Z}(F)$ of real zeros of $F(x)$ in $U=[0,1)$, taking into account their multiplicities, satisfies $\mathscr{Z}(F) \leq 2 n+n_{0}+2$; here $\operatorname{deg}(0)=-1$.

To prove Lemma 13, we need the following lemma and a known principle, the Derivation-division algorithm.

Lemma 14. For any $n \geq 0, m \geq 1$ and the real constants $\alpha$,

$$
\begin{aligned}
\mathscr{D}\left(p_{n}(x)(x+1)^{\alpha}\right) & =q_{n}(x)(x+1)^{\alpha-1}, \\
\mathscr{D}\left(p_{n}(x)\left(\frac{1+x}{1-x}\right)^{\alpha}\right) & =q_{n+1}(x) \frac{(1+x)^{\alpha-1}}{(1-x)^{\alpha+1}}, \\
\mathscr{D}^{m}\left(p_{n}(x)\left(\frac{1+x}{1-x}\right)^{\alpha}\right) & =q_{n+m}(x) \frac{(1+x)^{\alpha-m}}{(1-x)^{\alpha+m}} .
\end{aligned}
$$

In particular, when $m=n+1$, formula (69) becomes in the following form:

$$
\mathscr{D}^{n+1}\left(p_{n}(x)\left(\frac{1+x}{1-x}\right)^{\alpha}\right)=q_{n}(x) \frac{(1+x)^{\alpha-(n+1)}}{(1-x)^{\alpha+(n+1)}},
$$

where $p_{n}(x), q_{n}(x)$ are polynomials of degree $n, \mathscr{D}^{n}=\mathrm{d}^{n} / \mathrm{d} x^{n}$ with $n \geq 1$.

The previous lemma has been proved in [14] and [19]. Now, we will prove Lemma 13.

Proof. Differentiating $F(x)$ in formula (68) $n_{0}+1$ times, $\mathscr{D}^{n_{0}+1} P_{0}(x)=0$. According to Lemma 14 and dividing the expression $(1+x)^{-1 / 2-\left(n_{0}+1\right)}$, which does not vanish in $U=$ $[0,1)$, let $a=-1 / 2-\left(n_{0}+1\right)$; we can obtain

$$
\begin{aligned}
F_{1}(x) & \equiv(1+x)^{1 / 2+n_{0}+1} \mathscr{D}^{n_{0}+1} F(x) \\
& =P_{11}(x)+P_{12}(x)\left(\frac{1-x}{1+x}\right)^{a},
\end{aligned}
$$

where $P_{11}(x), P_{12}(x)$ are suitable polynomials of degree $n$. Applying Rolle's theorem, it follows that $\mathscr{Z}(F) \leq \mathscr{Z}\left(F_{1}\right)+$ $n_{0}+1$.

Differentiating $F_{1}(x)$ in formula $(71) n+1$ times and applying Lemma 14 again and dividing the expression (1 $x)^{a-(n+1)} /(1+x)^{a+(n+1)}$, which does not vanish in interval $U=[0,1)$, we have

$$
F_{2}(x) \equiv(1-x)^{-a+n+1}(1+x)^{a+n+1} \mathscr{D}^{n+1} F_{1}(x),
$$

where $F_{2}(x)$ is a polynomials of degree $n$. Therefore according to Rolle's theorem, taking into account their multiplicities, the total number $\mathscr{Z}(F)$ of real zeros of $F(x)$ in interval $U=[0,1)$ satisfies $\mathscr{Z}(F) \leq 2 n+n_{0}+2$. The proof is completed.

Now, we prove Theorem 2.

Proof. From Lemma 9, we have

$$
\begin{gathered}
\Phi(h)=\frac{1}{2}\left[\sum_{0 \leq k+j \leq n} a_{k, j}\left(\Phi_{k+1, j}^{1}+\Phi_{k+1, j}^{2}\right)\right. \\
\left.+\sum_{0 \leq k+j \leq n} b_{k, j}\left(\Phi_{k, j+1}^{1}+\Phi_{k, j+1}^{2}\right)\right] \\
=\frac{1}{2}\left[\sum_{\substack{0 \leq k+j \leq n \\
j \text { even }}} a_{k, j}\left(\Phi_{k+1, j}^{1}+\Phi_{k+1, j}^{2}\right)\right. \\
\left.+\sum_{\substack{0 \leq k+j \leq n \\
j \text { odd }}} b_{k, j}\left(\Phi_{k, j+1}^{1}+\Phi_{k, j+1}^{2}\right)\right] .
\end{gathered}
$$


By Lemmas 11 and 12, the previous formula becomes

$$
\begin{gathered}
\Phi(h)=\frac{1}{2}\left[\sum_{\substack{0 \leq k+j \leq n \\
k \text { odd } \\
\text { jeven }}} a_{k, j}\left(\Phi_{k+1, j}^{1}+\Phi_{k+1, j}^{2}\right)\right. \\
\left.+\sum_{\substack{0 \leq k+j \leq n \\
k \text { even } \\
j \text { odd }}} b_{k, j}\left(\Phi_{k, j+1}^{1}+\Phi_{k, j+1}^{2}\right)\right] .
\end{gathered}
$$

According to Lemma 10, we have that, if $j$ is even,

$$
\Phi_{k+1, j}^{\sigma}=\sum_{s=0}^{j / 2}(-1)^{s} h^{j / 2-s} C_{j / 2}^{s} \Phi_{k+1+2 s, 0}^{\sigma}
$$

if $j$ is odd,

$$
\Phi_{k, j+1}^{\sigma}=\sum_{s=0}^{(j+1) / 2}(-1)^{s} h^{(j+1) / 2-s} C_{(j+1) / 2}^{s} \Phi_{k+2 s, 0}^{\sigma}
$$

where $\sigma=1,2$.

According to (75) and (76), we can obtain that, if $k$ is odd, $j$ is even,

$$
\begin{aligned}
& \frac{1}{2}\left(\Phi_{k+1, j}^{1}+\Phi_{k+1, j}^{2}\right) \\
& =\sum_{s=0}^{j / 2}(-1)^{s} h^{j / 2-s} C_{j / 2}^{s} \\
& \times\left[\frac{\pi}{\sqrt{1-h}}+\frac{\pi i^{k+1+2 s}}{\sqrt{1+h}}\right. \\
& -\sum_{\substack{l=1 \\
l \equiv k+1+2 s(\bmod 2)}}^{k+1+2 s} 2^{l-k-1-2 s} \pi \\
& \cdot h^{(k+1+2 s-l) / 2} C_{k+1+2 s-l}^{(k+1+2 s-l) / 2} \\
& -\sum_{\substack{l=1 \\
l \equiv k+1+2 s(\bmod 2)}}^{k+1+2 s} 2^{l-k-1-2 s} \pi \\
& \left.\cdot h^{(k+1+2 s-l) / 2} i^{-k-1-2 s-l} C_{k+1+2 s-l}^{(k+1+2 s-l) / 2}\right]
\end{aligned}
$$

and if $k$ is even, $j$ is odd,

$$
\begin{aligned}
& \frac{1}{2}\left(\Phi_{k, j+1}^{1}+\Phi_{k, j+1}^{2}\right) \\
& =\sum_{s=0}^{(j+1) / 2}(-1)^{s} h^{(j+1) / 2-s} C_{(j+1) / 2}^{s}
\end{aligned}
$$

$$
\begin{gathered}
\times\left[\frac{\pi}{\sqrt{1-h}}+\frac{\pi i^{k+2 s}}{\sqrt{1+h}}\right. \\
-\sum_{l=1}^{k+2 s} 2^{l-k-2 s} \pi \\
\cdot h^{(k+2 s-l) / 2} C_{k+2 s-l}^{(k+2 s-l) / 2} \\
-\sum_{l \equiv k+2 s(\bmod 2)}^{k+2 s} 2^{l-k-2 s} \pi \\
\left.\cdot h^{(k+2 s-l) / 2} i^{-k-2 s-l} C_{k+2 s-l}^{(k+2 s-l) / 2}\right] .
\end{gathered}
$$

From (77) and (78), we have, if $k$ is odd, $j$ is even,

$$
\begin{aligned}
& \frac{1}{2}\left[a_{k, j}\left(\Phi_{k+1, j}^{1}+\Phi_{k+1, j}^{2}\right)+b_{k+1, j-1}\left(\Phi_{k+1, j}^{1}+\Phi_{k+1, j}^{2}\right)\right] \\
& =\left(a_{k, j}+b_{k+1, j-1}\right) \\
& \times \sum_{s=0}^{j / 2}(-1)^{s} h^{j / 2-s} C_{j / 2}^{s} \\
& \times\left[\frac{\pi}{\sqrt{1-h}}+\frac{\pi i^{k+1+2 s}}{\sqrt{1+h}}\right. \\
& -\sum_{\substack{l=1 \\
l \equiv k+1+2 s(\bmod 2)}}^{k+1+2 s} 2^{l-k-1-2 s} \pi \\
& \cdot h^{(k+1+2 s-l) / 2} C_{k+1+2 s-l}^{(k+1+2 s-l) / 2} \\
& -\sum_{\substack{l=1 \\
l \equiv k+1+2 s(\bmod 2)}}^{k+1+2 s} 2^{l-k-1-2 s} \pi \\
& \left.\cdot h^{(k+1+2 s-l) / 2} i^{-k-1-2 s-l} C_{k+1+2 s-l}^{(k+1+2 s-l) / 2}\right]
\end{aligned}
$$

where $k$ is odd, $j$ is even. 
According to (79), the Abelian integral $\Phi(h)$ of system (5) with $C(x, y)=1-x^{4}$ has the following form:

$$
\begin{aligned}
\Phi(h) & =\sum_{0 \leq k+j \leq n} \Phi_{k, j} \\
& =\sum_{\substack { u=1 \\
\begin{subarray}{c}{k+j=2 u-1 \\
\text { odd }{ u = 1 \\
\begin{subarray} { c } { k + j = 2 u - 1 \\
\text { odd } } }\end{subarray}}^{m} \Psi_{k, j}+\frac{1}{2} \sum_{\substack{j=1 \\
j \text { odd }}}^{n} b_{0, j}\left(\Phi_{0, j+1}^{1}+\Phi_{0, j+1}^{2}\right) \\
& =\sum_{u=0}^{m}\left(P_{u-1}(h)+\frac{Q_{u-1}(h)}{\sqrt{1-h}}+\frac{R_{u-1}(h)}{\sqrt{1+h}}\right) \\
& \equiv P_{m-1}(h)+\frac{Q_{m-1}(h)}{\sqrt{1-h}}+\frac{R_{m-1}(h)}{\sqrt{1+h}},
\end{aligned}
$$

where $P_{m}(h), Q_{m}(h)$, and $R_{m}(h)$ denote a polynomial of variable $h$ of degree $m$, whose coefficients are linear combinations of $a_{k, j}, b_{k, j}(0 \leq k+j \leq n)$.

According to Lemma 13, taking into account their multiplicities, the maximum number of real zeros of (81) in interval $U=[0,1)$ is $3 m-1$. From (73), (75), and (76), we know that $\Phi(0)=0$. Hence, $\Phi(h)$ has at least $3 m-2$ real zeros in the open interval $(0,1)$.

Applying the Poincaré-Pontryagin theorem, the upper bound of number of limit cycles for the system (5) with $C(x, y)=1-x^{4}$ is $3 m-2$. That is, the maximum number of limit cycles bifurcating from the period orbits of system (5) with $\varepsilon=0$ is $3[(n+1) / 2]-2$. The proof of Theorem 2 is completed.

\section{Acknowledgments}

The second author is partially supported by the National Natural Science Foundation of China, Grants no. 11171309 and no. 11172269, and the Zhejiang Provincial Natural Science Foundation of China Grant no. Y6110195.

\section{References}

[1] W. A. Coppel, "Some quadratic systems with at most one limit cycle," in Dynamics Reported, vol. 2, pp. 61-88, Wiley, Chichester, UK, 1989.

[2] X. D. Xie and S. L. Cai, "The planar quadratic system with an invariant parabola has at most one limit cycle," Chinese Science Bulletin, vol. 17, pp. 1540-1542, 1993.

[3] A. Zegeling and R. E. Kooij, "Uniqueness of limit cycles in polynomial systems with algebraic invariants," Bulletin of the Australian Mathematical Society, vol. 49, no. 1, pp. 7-20, 1994.

[4] S. L. Shui, "The planar quadratic system with an invariant cubic curve has at most one limit cycle," Acta Mathematicae Applicatae Sinica, vol. 24, no. 4, pp. 590-595, 2001.

[5] J. Llibre, R. Ramírez, and N. Sadovskaia, "On the 16th Hilbert problem for algebraic limit cycles," Journal of Differential Equations, vol. 248, no. 6, pp. 1401-1409, 2010.

[6] X. Zhang, "The 16th Hilbert problem on algebraic limit cycles," Journal of Differential Equations, vol. 251, no. 7, pp. 1778-1789, 2011.
[7] C. Christopher and C. Li, Limit Cycles of Differential Equations, Advanced Courses in Mathematics. CRM Barcelona, Birkhäuser, Basel, Switzerland, 2007.

[8] J. Llibre, J. S. Pérez del Río, and J. A. Rodríguez, "Averaging analysis of a perturbated quadratic center," Nonlinear Analysis: Theory, Methods \& Applications, vol. 46, no. 1, pp. 45-51, 2001.

[9] G. Xiang and M. Han, "Global bifurcation of limit cycles in a family of polynomial systems," Journal of Mathematical Analysis and Applications, vol. 295, no. 2, pp. 633-644, 2004.

[10] G. Xiang, M. Han, and T. Zhang, "The number of limit cycles for a family of polynomial systems," Computers \& Mathematics with Applications, vol. 49, no. 11-12, pp. 1669-1678, 2005.

[11] S. Li, Y. Zhao, and J. Li, "On the number of limit cycles of a perturbed cubic polynomial differential center," Journal of Mathematical Analysis and Applications, vol. 404, no. 2, pp. 212220, 2013.

[12] A. Buică and J. Llibre, "Limit cycles of a perturbed cubic polynomial differential center," Chaos, Solitons \& Fractals, vol. 32, no. 3, pp. 1059-1069, 2007.

[13] B. Coll, J. Llibre, and R. Prohens, "Limit cycles bifurcating from a perturbed quartic center," Chaos, Solitons \& Fractals, vol. 44, no. 4-5, pp. 317-334, 2011

[14] A. Gasull, J. T. Lázaro, and J. Torregrosa, "Upper bounds for the number of zeroes for some Abelian integrals," Nonlinear Analysis: Theory, Methods \& Applications, vol. 75, no. 13, pp. 5169-5179, 2012.

[15] A. Atabaigi, N. Nyamoradi, and H. R. Z. Zangeneh, "The number of limit cycles of a quintic polynomial system," Computers \& Mathematics with Applications, vol. 57, no. 4, pp. 677-684, 2009.

[16] A. Gasull, R. Prohens, and J. Torregrosa, "Bifurcation of limit cycles from a polynomial non-global center," Journal of Dynamics and Differential Equations, vol. 20, no. 4, pp. 945-960, 2008.

[17] H. Yao and M. Han, "The number of limit cycles of a class of polynomial differential systems," Nonlinear Analysis: Theory, Methods \& Applications, vol. 75, no. 1, pp. 341-357, 2012.

[18] G. Xiang and M. Han, "Global bifurcation of limit cycles in a family of multiparameter system," International Journal of Bifurcation and Chaos, vol. 14, no. 9, pp. 3325-3335, 2004.

[19] A. Gasull, J. T. Lázaro, and J. Torregrosa, "On the Chebyshev property for a new family of functions," Journal of Mathematical Analysis and Applications, vol. 387, no. 2, pp. 631-644, 2012. 


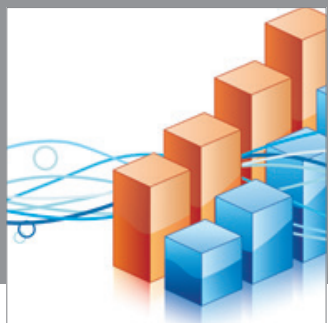

Advances in

Operations Research

mansans

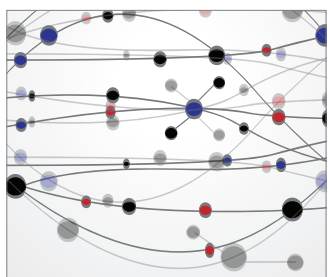

The Scientific World Journal
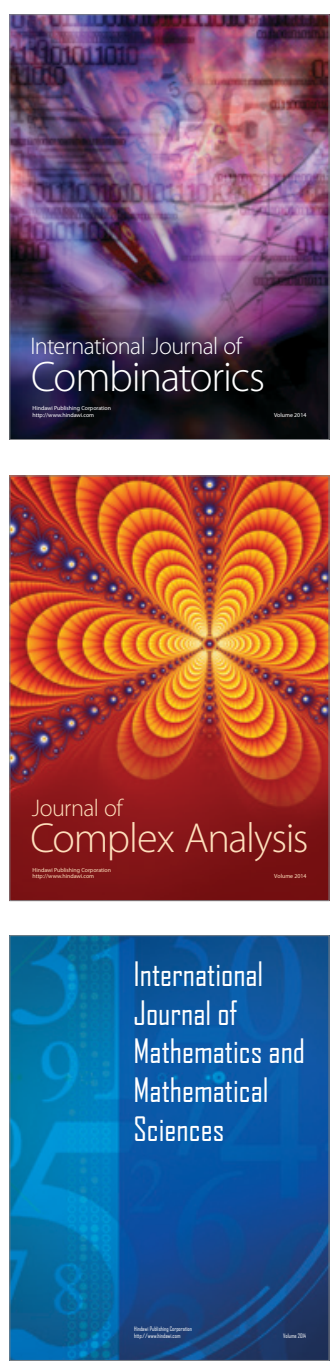
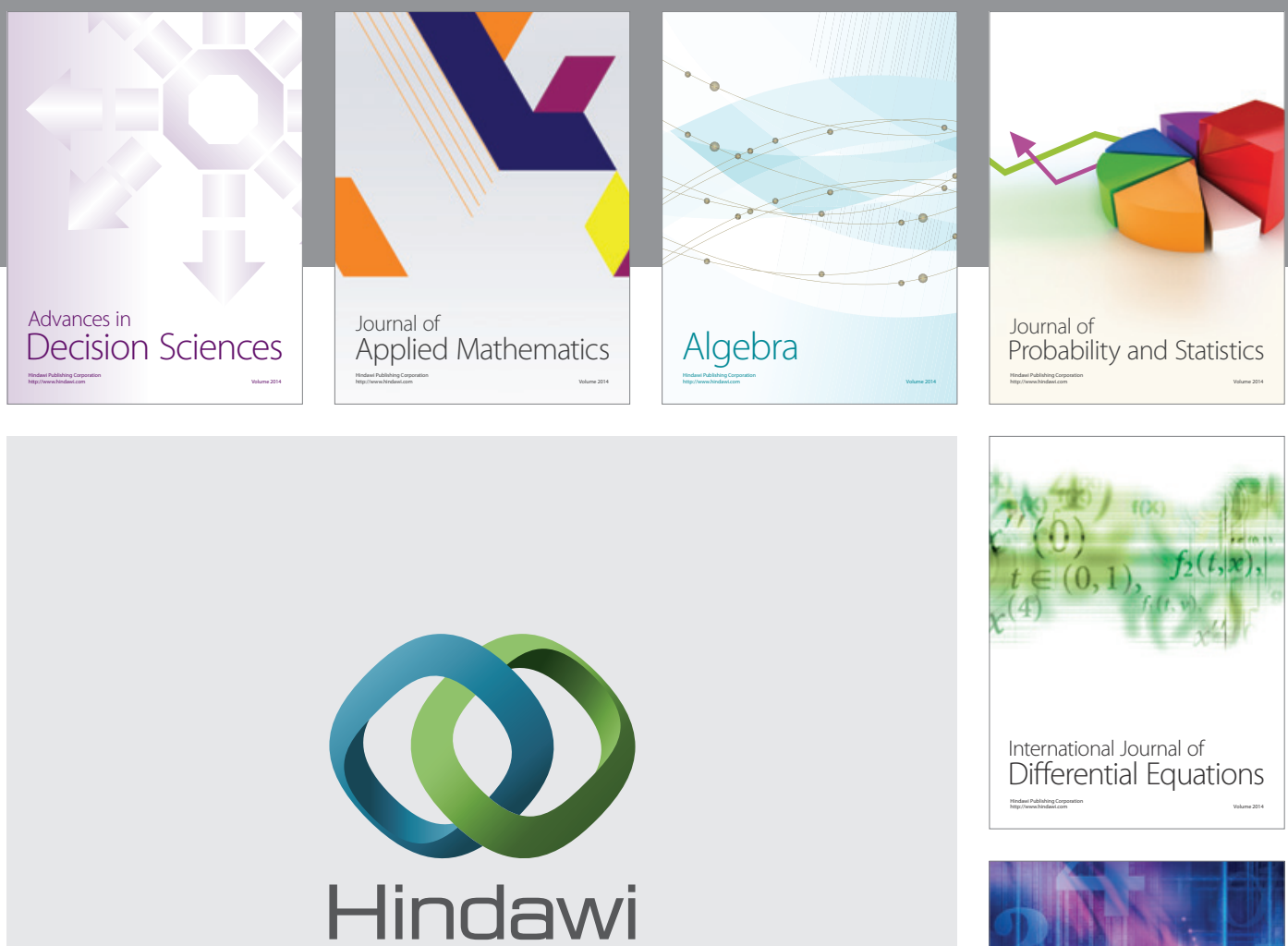

Submit your manuscripts at http://www.hindawi.com
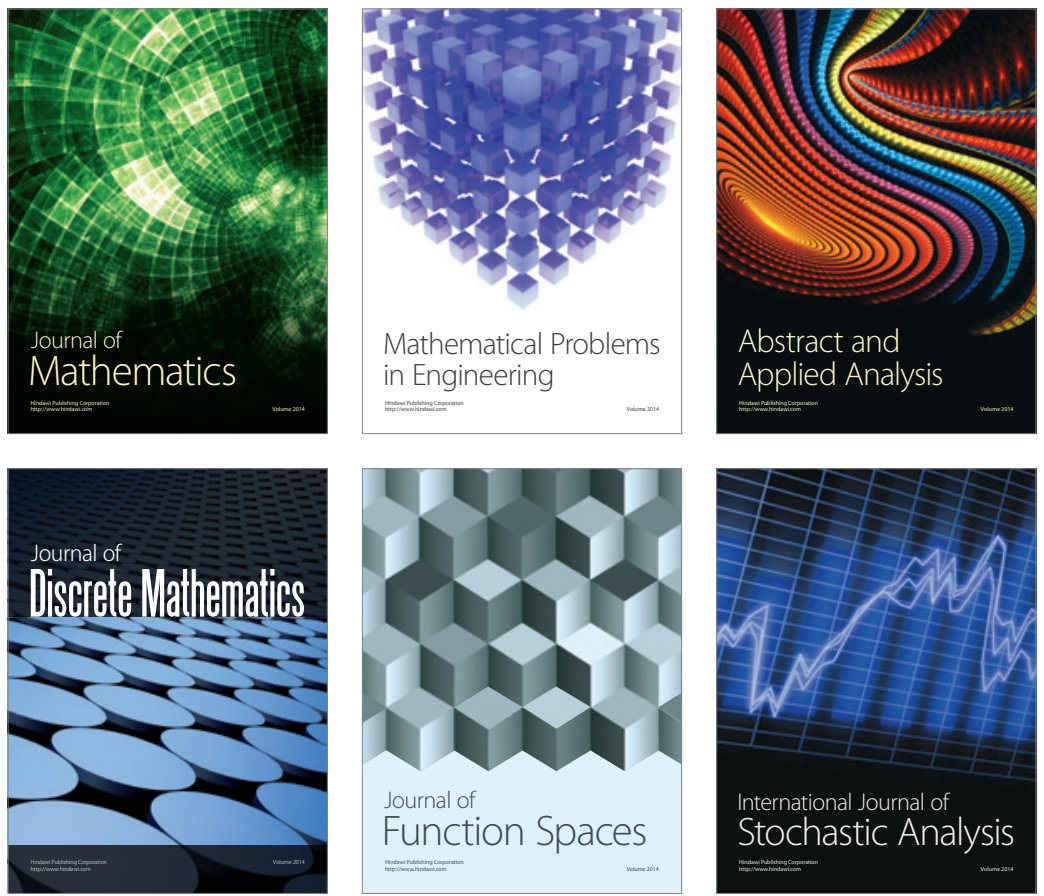

Journal of

Function Spaces

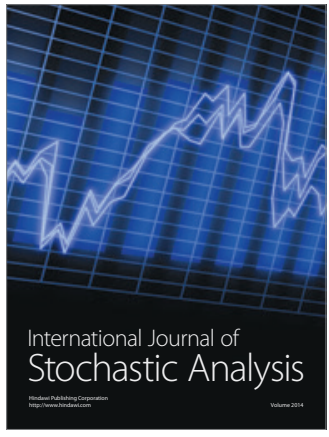

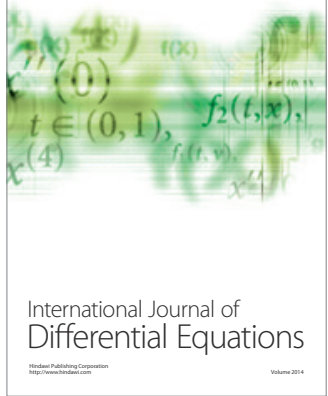
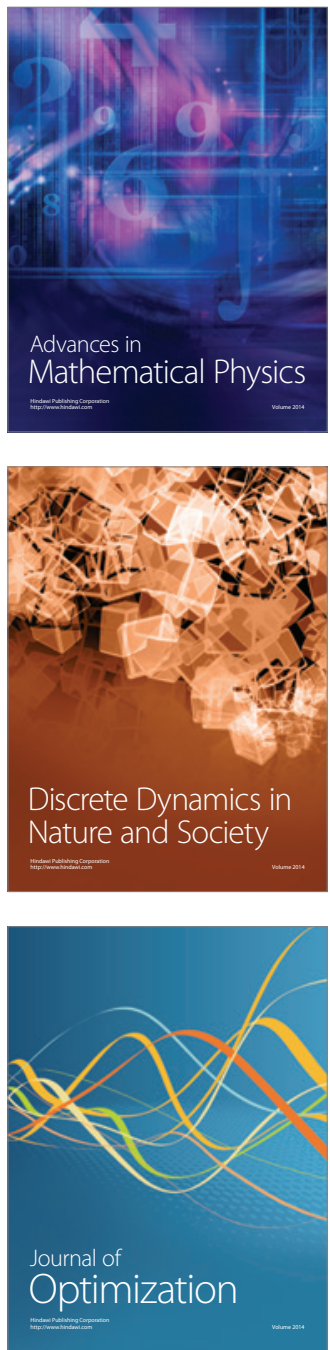